\title{
Design of charging station for electric vehicle batteries
}

\author{
Kusum $^{1}$, Chetan Parveer ${ }^{2}$ \\ ${ }^{1}$ Department of EE, CBS Group of Institution, Jhajjar, Haryana, India \\ ${ }^{2}$ Assistant Professor, Department of EE, CBS Group of Institution, Jhajjar, Haryana, India
}

\begin{abstract}
With the increasing requirement in green technologies in transportation, electric vehicles have proven to be the best short-term solution to reduce greenhouse gas emissions. The conventional vehicle drivers are still unwilling in using such a new technology, mainly because of the time duration (4-8 hours) required to charge the electric vehicle batteries with the currently existing Level I and II charging station. For this reason, Level III fast- charging stations capable of reducing the charging duration to 10-15 minutes are being designed and developed. The present thesis focuses on the design of a fast-charging station for electric vehicle, in addition to the electrical grid, two stationary energy storage devices flywheel energy storage and a super capacitor is being used. Power electronic converters used for the interface of the energy sources with the charging stations are designed. The design development also focuses on the energy management that will minimize the battery charging time. For this reason, an algorithm that minimizes durations with its mathematical formulation is required, and its application in fast charging will be illustrated.
\end{abstract}

Keywords-charging station, electric vehicle, batteries.

\section{INTRODUCTION}

This chapter gives a background of the thesis topic: Design for Fast Charging Station for Electric vehicle Batteries then summarizes the work that has been conducted previously in fast charging technology. The problem is then explained, and the thesis outline is provided.

\section{Background}

This section defines what a PHEV is and describes briefly the equipment involved to charge it.

\section{PHEV Definition}

A Plug-in-Hybrid Electric Vehicle (PHEV) is a hybrid automobile with a higher-capacity battery that can be recharged by connecting the vehicle to the electrical network [1]. When the battery is below $20 \%$ capacity, a conventional combustion engine takes over and offers to the driver the same autonomy as a conventional vehicle. The PHEV has been recognized as the best short-term, economically viable opportunity for significantly reducing oil dependency and $\mathrm{CO}_{2}$ emissions without altering motorists' driving behaviour [1]. Consequently, nearly all major car makers have invested significant resources in PHEV development, and Toyota and GM delivered their first PHEVs (in small numbers) in 2010.

\section{Flywheels}

Flywheel energy storage (FES) is an electromechanical device that stores energy in kinetic form in a rotating mass [49]. Flywheels are useful when there exists an imbalance between the generated power and the power demanded by the load [56]. In such devices, the charging and discharging processes are done by varying the rotational velocity of the mass: to store some electricity, a motor converts the external electrical energy into mechanical energy (charging), on the other hand to deliver some energy the motor acts as a generator and converts the energy into electrical form (discharging) [56]. Some control strategies have been found to apply flywheels in EVs. One of them has been found in [57], where the charging process is done using fuzzy logic and a PI controller whereas the discharging is done by simply applying the PWM strategy to the interfacing converter (AC/DC) [57].

FESs have been recognized as being the cleanest energy storage devices [59] and find their applications in the following areas:

- Previously, FESs have always been used for short-term energy storage in rotating machines and engines to deliver smooth power [58].

- Recently, they are being used for electrical energy storage [60]. In such case the FES is referred to as a mechanical battery energy storage device: it always stores kinetic energy and releases it in electrical form upon demand. This last advantage will be considered largely in this thesis.

- For the next few years, researches are being conducted in order to design higher specific power density $(\mathrm{kW} / \mathrm{Kg})$ 
and higher specific energy $(\mathrm{kWh} / \mathrm{Kg})$ density. The first one depends completely on the motor/generator that drives it [60].
Any FES is composed of four major parts: a rotor, a rotor bearing, a container, and a power interface [59]. Figure below displays the main flywheel parts.

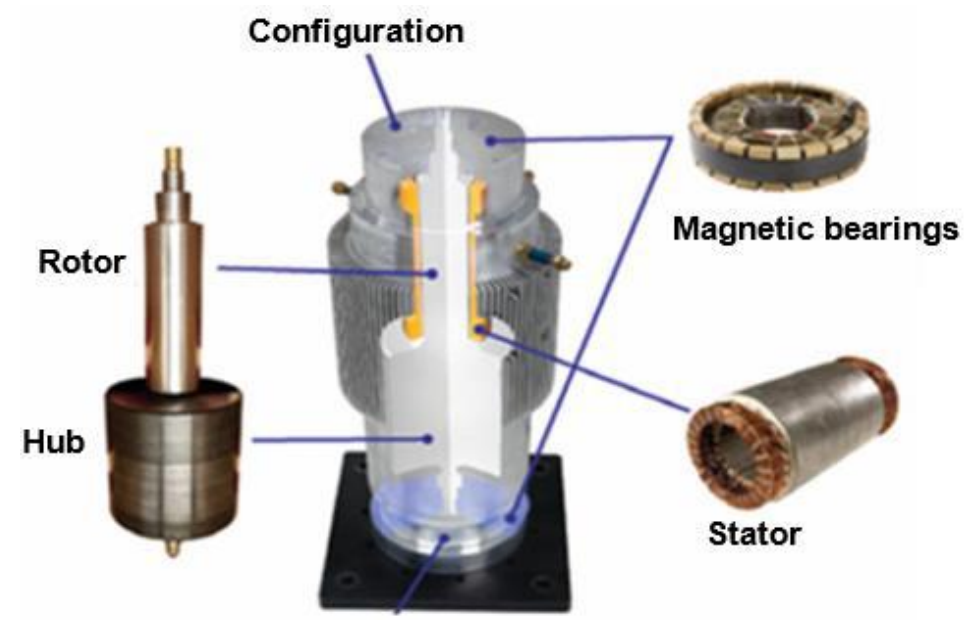

Housing

Fig.1: Main FES Components [59]

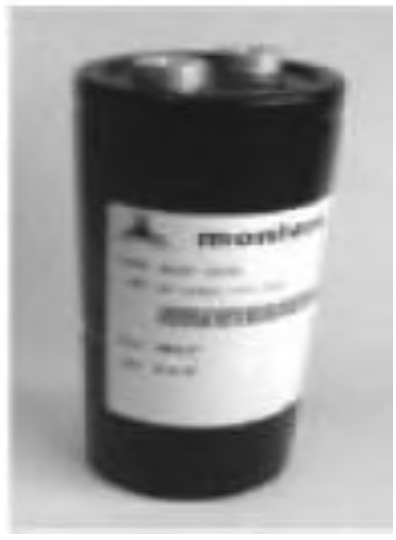

Fig.1.1: A Typical Supercapacitor [63]

The steady-state energy losses in flywheels are mainly due to the drag forced that is induced by the magnetic field of the superconductor magnetic bearing and permanent magnet (PM)-type motor/generator (PMSM/G) [61]. However, the largest losses occur from the PMSM/G, and an acceptable solution would be to simultaneously rotate the PMSM/G and the PM [62].

\section{Supercapacitors}

Also known as electric double layer capacitors (EDLC) or ultracapacitors, such devices behave exactly like any normal capacitor with the differences of having a much higher capacitance (in the order ranging from tens to hundreds of Farads) and a higher power density which lets them charge and discharge rapidly [63] and allows them to be used in applications to replace batteries. A typical supercapacitor is shown in Figure below.

Such devices store energy using the following mathematical relation that relates the energy stored $\mathrm{E}$ (in $\mathrm{J}$ ) to the capacitance $\mathrm{C}$ (in F) and the voltage across it $\mathrm{U}$ (in V) [50]:

$$
\mathrm{E}=\mathrm{C} \cdot \mathrm{U}^{2}
$$

They are clas sified into three categories [64]:

- Double-layer capacitors depend on the double electric layer mechanism.

- Electrochemical capacitors rely on the fast Faraday oxide-reduction reactions.

- Hybrid capacitors are a combination of the two previous categories.

A control strategy has been proposed for the supercapacitors 
to support current peaks that are momentarily demanded by electrical road vehicles. Such a method is since the supercapacitors must be discharged once the current demanded by the load becomes greater than the reference limit current for battery discharge [65]. Supercapacitors are safe, possess an average light weight, can be recycled, and are environmentally friendly [66], which is what makes them mostly popular in the domain of energy storage for traction applications [67] and elevator systems with a soft commutated interface [64]. Additional applications include the following:

- Diesel-electric locomotives power assistance:

supercapacitors are preferred compared to

electromechanical accumulators when being used in

power assistance [68]

- Recuperation of the braking energy that is wasted in the braking resistors [69].

- Sole energy storage device in hybrid electric cars if they are dimensioned appropriately.

Despite the benefits just mentioned, the energy that can be stored in a supercapacitor is low, which prevents large vehicle autonomy. For this reason, some methods have been found in order to allow fast energy transfer between super capacitors in transport applications, such as the introduction of sequential supply [70].

\section{PROBLEM STATEMENT}

Fast charging of PHEV batteries means that the duration required to charge such batteries must be minimized, which implies the use of the grid and additional sources of energy that must be managed efficiently and intelligently.

A waiting period is also required to recharge the storage devices once the PHEV leaves the station. Such a period should also be minimized in order to reduce the time that the client needs to wait at the station before charging the battery, and to accelerate the battery swapping process at some charging stations if applicable.

Finally, it is important to look at some aspects of the impacts caused by such charging stations on the grid such as harmonics, THD, phase unbalance, power factor, ground fault and electricity generation.

s mentioned in Section 1.4, the charging station is designed to charge PHEV batteries whose energy capacities do not exceed $15 \mathrm{kWh}$, from a minimum of $20 \%$ of the battery state of charge (SOC) to a maximum of $95 \%$ of the battery SOC. This implies that the maximum energy output by the charger will be:

$\mathrm{E}_{0, \max }=\mathrm{E}_{\mathrm{PHEVmax}} \cdot\left(\mathrm{SOC}_{\max }-\mathrm{SOC}_{\min }\right)=15 \cdot(0.95-0.20)$

$\mathrm{E}_{\mathrm{O}, \max }=11.25 \mathrm{kWh}$

Using (2.1), (2.2), and (2.3), the maximum energy provided by the charging station storage devices, EStorage,max, can be found as follow:

$$
\begin{array}{r}
\text { EStorage, } \max =\mathrm{EO}_{\text {, } m a x}-\text { EGrid }=11.25-5 \\
\text { EStorage, } \max =6.25 \mathrm{kWh}
\end{array}
$$

The energy management in the designed charging station is summarized in Table 2.3 below.

Table.2.3: PHEV Charging Station Energy Management

\begin{tabular}{|c|c|c|c|}
\hline Sources & Grid & Storage Devices & Output \\
\hline Energy $(\boldsymbol{k W h})$ & 5 & 6.25 & 11.25 \\
\hline
\end{tabular}

The choice of the charger storage devices is developed in the next section.

\section{Station Storage Devices Choice}

The present section lists the most popular stationary energy storage devices performance requirements, and then justifies the charging station devices choice.

\section{Performance Requirements}

The chosen energy storage devices must ideally satisfy all of the following performance criteria in order to maximize the fast charging station efficiency:

- Dynamicity: The charging station is designed to charge a battery in a maximum 15 minutes (short duration). The storage device must thus be able to charge and discharge in this period.

- High Power Density: The device must be able to deliver a high amount of power in a short period of time.

- High Efficiency: The charging station must meet its maximu $\mathrm{m}$ possible efficiency. This last criterion depends on the main station parts: converters, storage devices, etc. Therefore, it is a must to consider the storage devices that have the highest efficiency.

- Environmentally Friendly: The device must have no or negligible negative impacts on the environment.

Table 2.4 below displays the most popular stationary storage devices according to the previously mentioned criteria [71, 72]. 
Table.2.4: Classification by Criterion

\begin{tabular}{|l|c|c|c|c|}
\hline Storage Technology & ife time (cycles) & Power Density & iency (\%) & $\begin{array}{l}\text { Impact on the } \\
\text { Environment }\end{array}$ \\
\hline Flow Batteries & $1500-2500$ & Low & $75-85$ & Medium \\
\hline Metal-Air Batteries & $100-200$ & Low & 50 & Medium \\
\hline NAS Batteries & $2000-3000$ & Low & 89 & High \\
\hline Lead-Acid Batteries & $200-300$ & Medium & 75 & High \\
\hline Li-Ion Batteries & $300-500$ & Medium & 95 & Medium \\
\hline Supercapacitors & $10000-100000$ & High & $93-98$ & Low \\
\hline Flywheels (FES) & $10^{5}-10^{7}$ & High & 90 & Low \\
\hline
\end{tabular}

\section{STATIC POWER SWITCHES}

This section covers the existing technologies of static power electronic switches and then justifies the use of IGBT as the best option for the design of the converters.

\section{i. Existing Technologies}

The most popular controllable static switches used in the design of the power electronic convert ers of Table 1.1 are displayed below in Table 3.1 [19].

Table.3.1: Main Controllable Switches Comparison

\begin{tabular}{|c|c|c|}
\hline Device & Power Capability & Switching Speed \\
\hline MOSFET & Low & Fast \\
\hline IGCT & High & Slow \\
\hline IGBT & Medium & Medium \\
\hline
\end{tabular}

Furthermore, the grid outputs a real power of $30 \mathrm{~kW}$ (see Section 2.2) and has a frequency of $60 \mathrm{~Hz}$; both are in the medium range of operation. In the technologies of Table 3.1, the IGBT tends to be the best option for the design of the charging station is power electronic interfaces. An IGBT converter has an efficiency of typically $90 \%$ [58].

\section{Converter Design}

In order to turn on and off the controllable switches inverters and rectifiers, many types of gating signals can be used, among them are [19]:

- Square wave inverters: DC input must be varied to control the magnitude of the output AC voltage.

- Voltage cancellation: Switches operate at 0.5 duty cycle while the DC input remains fixed.

- Pulse-Width-Modulation (PWM): A modulating signal where the $\mathrm{AC}$ side frequency is compared with a carrier having a frequency such that the frequency modulation $\mathrm{m}_{\mathrm{f}}$ (defined below) is a large odd integer:

The most popular gating generation used in inverters is a sinusoidal PWM, where the control signal is a sine wave; it generates harmonic voltages in the range of the switching frequency and higher, which can be easily filtered out [19].
The grid-side converter is shown in Figure 3.1.

Figure Grid-side Converter

\section{Flywheel Energy Storage (FES) Converter}

When acting as a generator, a FES converts kinetic energy into electrical energy [58]. This can be translated in the following way: when rotating at an angular speed $\omega(\mathrm{rad} / \mathrm{s})$, the energy is converted into AC currents that must be converted to DC currents via an AC/DC converter [81].

\section{Supercapacitor Converter}

Like any conventionalcapacitor, a supercapacitor charges and discharges in a DC environment [66]. The interface here is thus between DC quantities, and a bidirectional chopper is required to allow both charging and discharging of the supercapacitor.

\section{Charging Station Supercapacitor}

The supercapacitor is required to provide the battery with $10 \%$ of its required energy during the beginning of the battery charging process (see Chapter 5 ). Thus, the maximu m output energy provided by the supercapacitor is found as follows: 
EScap, $\max =0.1 \cdot \mathrm{E}_{, \max }=0.1 \times 11.25=1.125 \mathrm{kWh}$

However, since the supercapacitor is interfaced with the charging station dc bus via an IGBT converter, the efficiency will play a role:

The relation relating the supercapacitor moment capacitance to its rated voltage (see Section 1.2.10, equation (1.8)) to provide the energy $\mathrm{E}_{\text {Scap,in }}$ is reminded below:

Table.3.6: Supercapacitor Options (for $E_{\text {Scap, in }}=1.25 \mathrm{kWh}$ )

\begin{tabular}{|l|c|c|c|c|c|}
\hline Capacitance $(\boldsymbol{F})$ & 100 & 150 & 200 & 250 & 300 \\
\hline Rated Voltage $(\boldsymbol{V})$ & 300 & 245 & 212.13 & 190 & 173.20 \\
\hline
\end{tabular}

An acceptable option would be a series / parallel combination of supercapacitors whose resulting capacitance and voltage are $150 \mathrm{~F}$ and $245 \mathrm{~V}$, respectively.

\section{Electrical Specifications}

The output converter input current is equal to the sum of the output currents of the three previously mentioned converters:

$$
\begin{gathered}
\text { Itotal,DC }=\text { IGrid,DC + IFES,DC + IScap, DC } \\
\mathrm{I}_{\text {total,DC }}=50+51.25+22.5=123.75 \mathrm{~A}
\end{gathered}
$$

The converter input power is calculated as follows:
$\mathrm{P}_{\text {total }, \text { in }}=\mathrm{V}_{\text {DC. }}$ Itotal, $\mathrm{DC}=600 \times 123.75=74.25 \mathrm{~kW}$

The converter output power is calculated as follows:

The converter output voltage is the maximu $\mathrm{m}$ PHEV battery voltage, which is $270 \mathrm{~V}$ (Table 2.1).

Finally, the converter output current is calculated as follows:

With the information (3.13) -(3.16), the following Table 3.8 which displays the charging station output converter electrical specification can be drawn.

Table.3.8: Charging Station Output Converter Electrical Specifications

\begin{tabular}{|l|c|c|}
\hline & Input Side (DC Value) & Output Side (DC Value) \\
\hline Voltage $(\boldsymbol{V})$ & 600 & 270 \\
\hline Current $(A)$ & 123.75 & 67 \\
\hline Power $(k \boldsymbol{W})$ & 74.25 & 67 \\
\hline
\end{tabular}

\section{Complete Power Circuit}

The present section describes the combined design of the charging station, and then lists its electrical specifications.

\section{Circuit Design}

The electrical grid, the supercapacitor, and the FES are all interfaced via their respective power electronic converters previously designed to a common dc bus. The interface with the PHEV battery is also done via a bidirectional DC/DC converter (see Section 3.5).

\section{Electrical Specifications}

The energy and power requirements of each current source are summarized in Table 3.9 below:

Table.3.9: Charging Station Electrical Specifications

\begin{tabular}{|l|c|c|c|}
\hline & Electrical Grid & FES & Supercapacitor \\
\hline $\begin{array}{l}\text { Maximum } \\
\text { output energy }(\boldsymbol{k W h})\end{array}$ & 5.56 & 5.70 & 1.25 \\
\hline Converter Efficiency $(\%)$ & 0.9 & 0.9 & 0.9 \\
\hline $\begin{array}{l}\text { Maximum } \\
\text { output energy }(\boldsymbol{k W h})\end{array}$ & 5 & 5.125 & 1.125 \\
\hline $\begin{array}{l}\text { Maximum time of charging } \\
\text { operation (min) }\end{array}$ & 10 & 10 & 5 \\
\hline Maximum Output power $(\boldsymbol{k W})$ & 30 & 30.75 & 13.5 \\
\hline
\end{tabular}


Figure below displays the combined charging station power circuit.

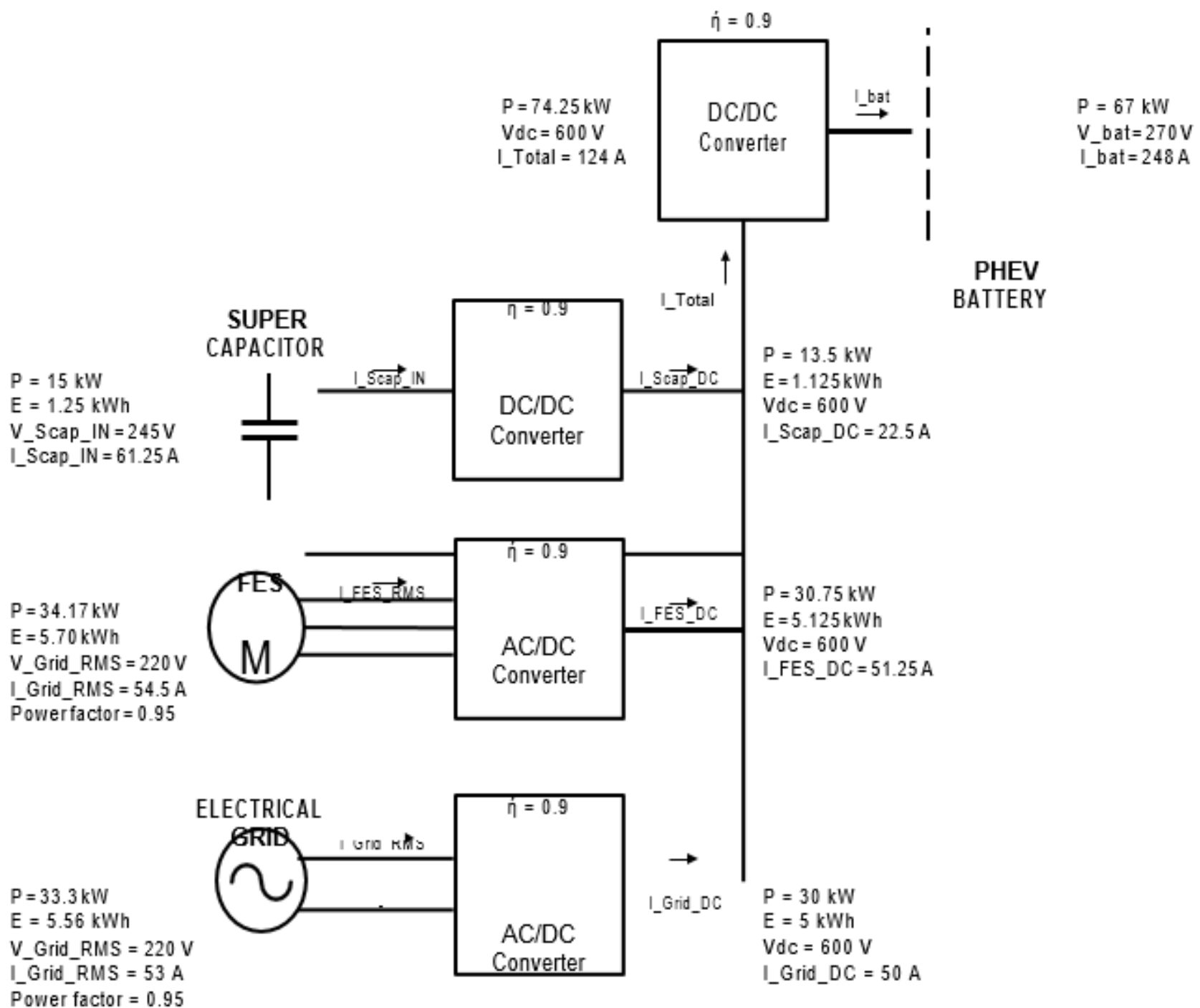

Fig.3: Charging Station Power Circuit

Charging Station Control Circuit

In the previous chapter, the power circuit has been designed along with its electrical specifications. The present chapter explains the control of each individual converter, then the whole charging station control circuit.

\section{CONVERTERS INDIVIDUAL CONTROL}

This section elaborates on the individual control schemes of each of the four-charging station power electronic converters.

\section{FES Control}

The FES control by itself is difficult.

\section{FES Emulation}

It has been demonstrated that a FES could easily be emulated by a PMDC (Permanent Magnet DC Machine) [83]. Such operation would considerably decrease the system size and cost [83]. The FES system model will thus be replaced by a DC machine model as shown in Figure 


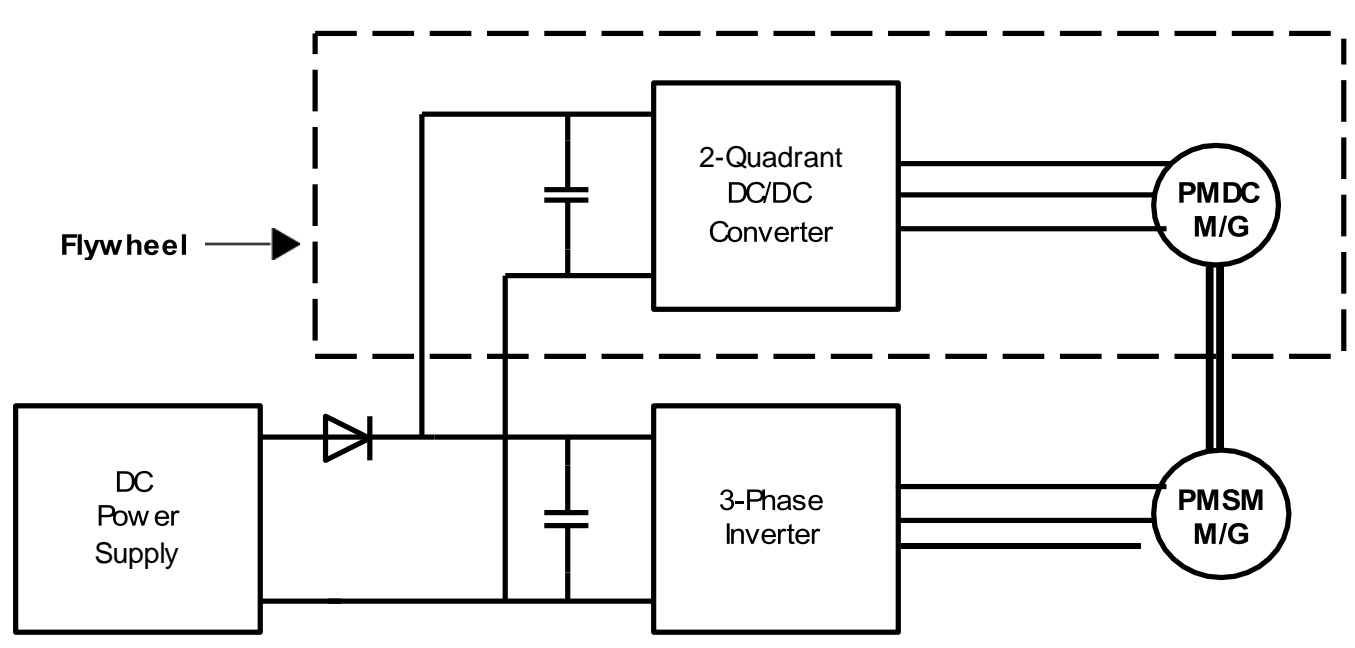

Fig.4: Flywheel Emulation Using a PMDC [Based on 83]

The power flow in the above system is bidirectional. Every power transfer is done through the permanent magnet synchronous machine (PMSM), which can act as motor and generator. The DC power supply not only imposes the dc bus voltage, but it also compensates for the systemlosses.

It is reminded that the kinetic energy $d W$ (in $\mathrm{J}$ ) stored in the above system with moment of inertia $J_{F}$ (in $\mathrm{Kg} . \mathrm{m}^{2}$ ) and rotating from one speed $\omega_{1}$ to another speed $\omega_{2}$ (in $\mathrm{rad} / \mathrm{s}$ ) is expressed as [83]:

$$
d W=-\cdot \mathrm{J}_{\mathrm{F}} \cdot\left(\omega_{2}{ }^{2}-\omega^{2}\right)
$$

The above system is designed to operate in three modes based on the stored energy using the above formula: charging, discharging, and no charging [83]. Each model is now explained below.

In the charging mode, the power flows from the dc bus to the PMDC through the PMSM. In such a case the DC machine is accelerated from the speed $\omega_{1}$ to a higher speed $\omega_{2}$. In the discharging mode, the power flows from the PMDC to the dc bus through the PMSM. In such a case, the DC machine is decelerated from the speed $\omega 2$ to a lower speed $\omega_{1}$.

In the no charging mode, the DC machine runs at a constant at speed, and there is thus no power flow.

\section{System Control}

The amount of energy transferred in or out of the flywheel can be controlled by controlling the PMSM torque by imposing either a positive or negative torque command in the PMSM controller, which is based on field orientated control
(FOC) in a rotor frame.

Under ideal FOC, $i$ is set to 0 and the PMSM electromagnetic torque $\tau$ can then be written as [84]:

where $\lambda_{\text {af }}$ is the rotor flux linkages, $\mathrm{i}^{\mathrm{r}}$ is the torque component of the stator current in the rotor reference frame, and $p$ is the number of pole pairs.

The relationship between $\mathrm{i}^{\mathrm{r}}$ and $\mathrm{i}$ (Flywheel transfer current to/from the inverter) is found from the steady-state power balance between the dc power going into the inverter and the ac power going into the PMSM [83]. If we neglect the inverter losses, we have:

$$
\mathrm{P}_{\text {inv }}=\mathrm{PSM}_{\mathrm{SM}}
$$

$$
\mathrm{FW} \cdot \mathrm{VDC}=\tau_{\mathrm{E} \_\mathrm{SM}} \cdot \omega_{\mathrm{m}}(4.2)
$$

Substituting the electromagnetic torque expression (4.1) into the power balance relation (4.2) and solving for $\mathrm{i}^{\mathrm{r}}$ we get the following relation (which is also the flywheel control algorithm for both charging and discharging modes):

Figure below displays the FES control loop according to the above relation. At start-up, the control algorithm starts the PMSM using an initiation algorithm. The PI current controller is used to maintain the power flow. 


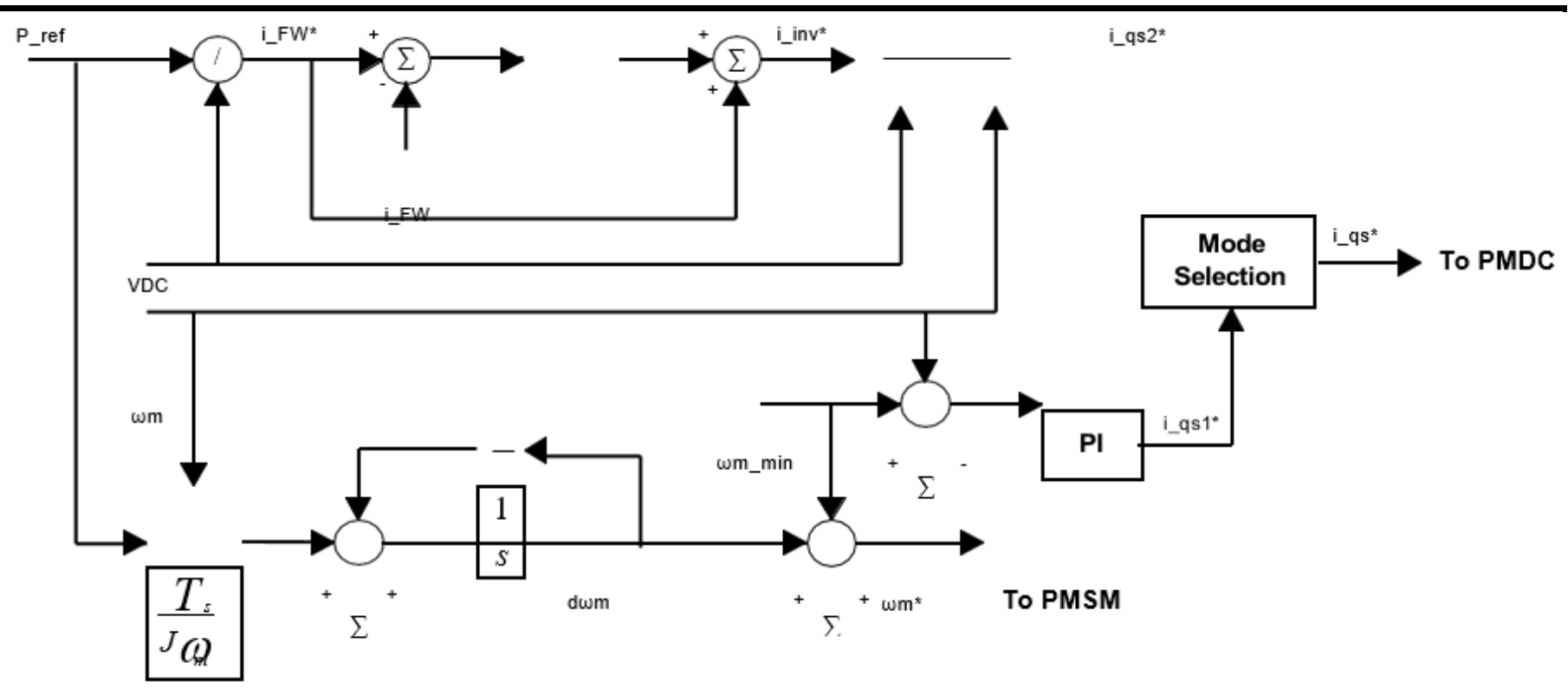

Fig.5: Flywheel Charging and Discharging Control [Based on 83]

\section{Supercapacitor Converter Control}

The supercapacitor control design is analogous to the flywheel control design shown in Figure 4.3 above and is designed to allow charging and discharging of the super capacitor. The DC/DC converter output current reference is produced by dividing the reference supercapacitor's required power by the dc bus voltage. The error in current is then fed to a PI current regulator in order to maintain the power flow between the charger dc bus and the supercapacitor. Finally, the regulator output will be directed to the supercapacitor control scheme. The mode of operation (charging or discharging mode) depends on the sign of the supercapacitor's reference power.

\subsubsection{Charging Station Output Converter Control}

The control scheme of the output converter is very similar to the grid converter control and is presented in Figure below. It is composed of two nested control loops:

The outer control loop is designed for voltage regulation. The error between the battery nominal reference voltage and the measured battery charging voltage is fed to a PI controller, whose output is the battery charging current reference.

The inner loop regulates the battery charging current. The reference here is the output of the previous outer loop. The error between the reference and the measured current is also fed to a PI controller whose output is sent to the charging station output converter control scheme.

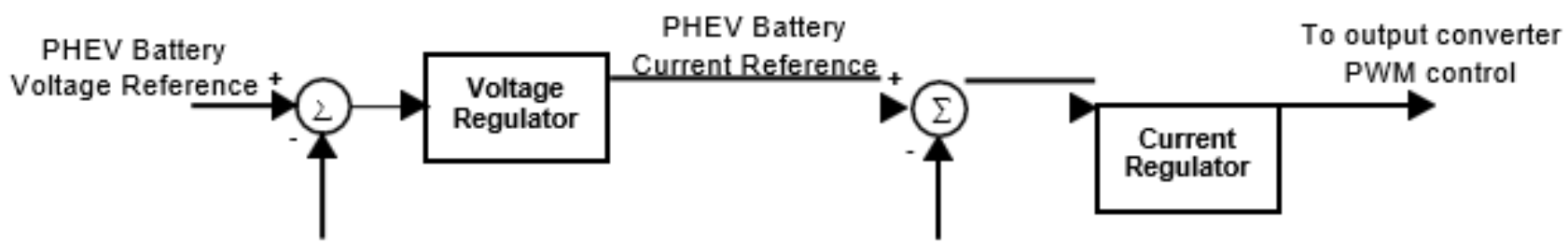

Measured PHEV
Battery Voltage

\section{Measured PHEV}

Battery Current

Fig.6: Charging Station Output Converter Control

\section{Control Circuit Design}

This section presents an algorithm that combines the previous individual controls in such that it minimizes the PHEV battery charging duration.

\section{Charging Station Central Control}

A charging station cycle is composed of a PHEV battery charging period (two phases) that does not exceed 15 minutes, followed by a period (one phase) during which the storage devices are fully recharged, which lasts a maximum of 7.5 minutes. This is demonstrated in Figure. 


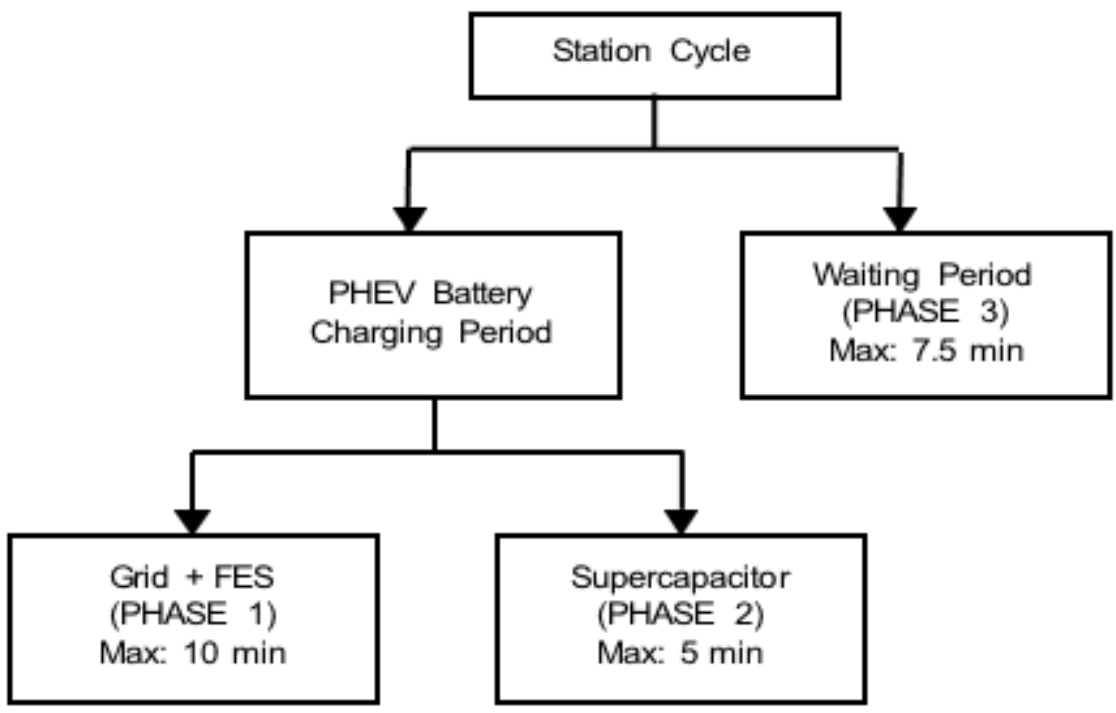

Fig.7: Schematic Diagram of the Charging Station Cycles

More details on the three phases will be provided in the next chapter.

\section{Central Control Algorithm}

In order to obtain the requirements of Figure, the algorithm of
Figure below is proposed. Table 4.1 displays the abbreviations used in the flowchart in Figure. Blue and red instructions indicate whether energy is being transferred from the charger to the battery, or from the grid to the storage devices (to recharge them), respectively.

Table.4.1: Figure 7 Abbreviations

\begin{tabular}{|l|c|c|c|c|}
\hline Abbreviations & SC & FES & C_act & C \\
\hline Expressions & supercapacitor & flywheel & $\begin{array}{c}\text { Actual battery } \\
\text { capacity }\end{array}$ & $\begin{array}{c}\text { Total battery } \\
\text { capacity }\end{array}$ \\
\hline
\end{tabular}

In standby mode, there is no PHEV battery connected to the charging station, and the energy storage devices have been fully recharged. At this moment, the FES rotates at constant speed $\Omega_{\mathrm{o}}$ (see Section 4.1.2) and thus there is no power transfer
$(\mathrm{dW}=0)$. The supercapacitor voltage continues to increase asymptotically to its rated voltage, whereas its current tends asymptotically to 0 . The charging station remains in this mode until the arrival of a PHEV at the station. 


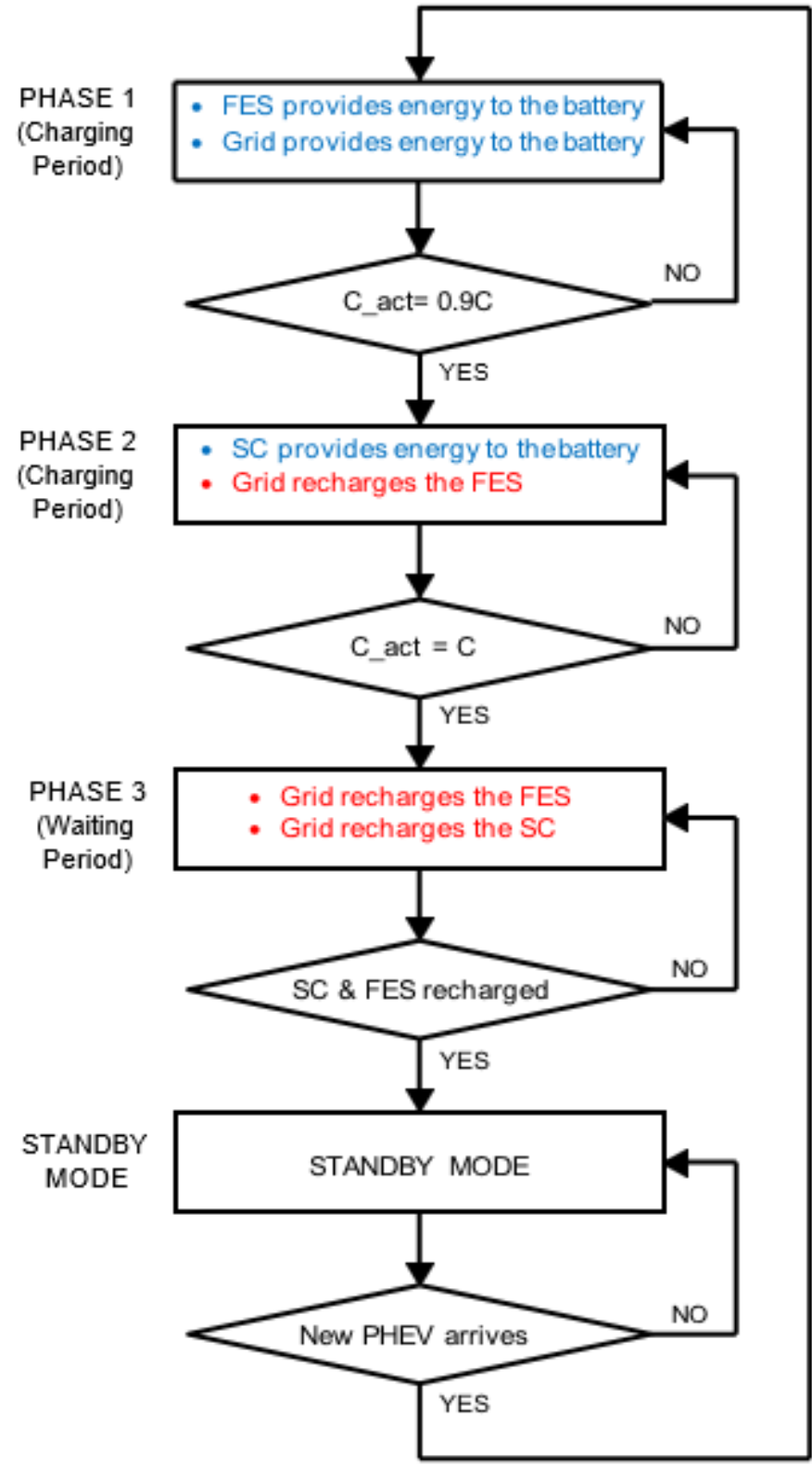

Fig.8: Central Control Algorithm Flowchart

\section{CHARGING STATION OPERATION}

The charging station operates as described in the flowchart shown in Figure. The energy management of the energy sources is done via optimization; the topic of this chapter. Two examples will be used to illustrate such operation.

\section{Charging Time Minimization}

The charging station is designed to minimize the PHEV battery charging time and required duration to recharge the storage devices. This requires an effective management strategy of the charging stations' energy sources: grid, supercapacitor, and FES.

\section{Charging Station Cycle}

As mentioned previously, a charging station cycle is composed of three phases. The order of Phases 1 and 2 has been established by considering the fact that some PHEV users may have a limited amount of time to spend at the charging station; for this reason, most of the PHEV battery charging is done in the beginning of the cycle (Phase 1). The maximu $\mathrm{m}$ duration of each phase has been determined by optimization (further details on the order of the charging durations of the FES and supercapacitor were provided in section 2.3.2).

- Phase 1: The FES and the electrical grid provide energy 
to the PHEV battery until it reaches $90 \%$ of its required capacity. The maximum duration of this phase is 10 minutes.

- Phase 2: While the supercapacitor provides energy to the PHEV battery until it reaches its required capacity, the electrical grid is recharging the FES with a capacity determined by optimization (described in Section 5.1.3). The maximum duration of this phase is 5 minutes.

- Phase 3: During this phase, which lasts no more than 7.5 minutes, the electrical grid is recharging the supercapacitor and the FES to their respective full capacities. It is also called the "waiting period" because, during this time, no PHEV battery is allowed to be connected to the charging station.

Once the storage devices are fully recharged, the charging station enters its standby mode until anotherPHEV arrives at the charging station to recharge its battery. Examples of the systemoperation are provided in Section 5.2.

\section{Problem Formulation}

A charging station cycle is graphically represented in Figure, where:

- Blue and red colours indicate whether energy is being transferred from the charger to the battery, or from the grid to the storage devices (to recharge them), respectively.

- Positive and negative quantities indicate whether energy is being delivered or absorbed by the device, respectively.

- $\quad \mathrm{P}$ and $\mathrm{C}$ denote the grid power and the PHEV battery capacity, respectively.

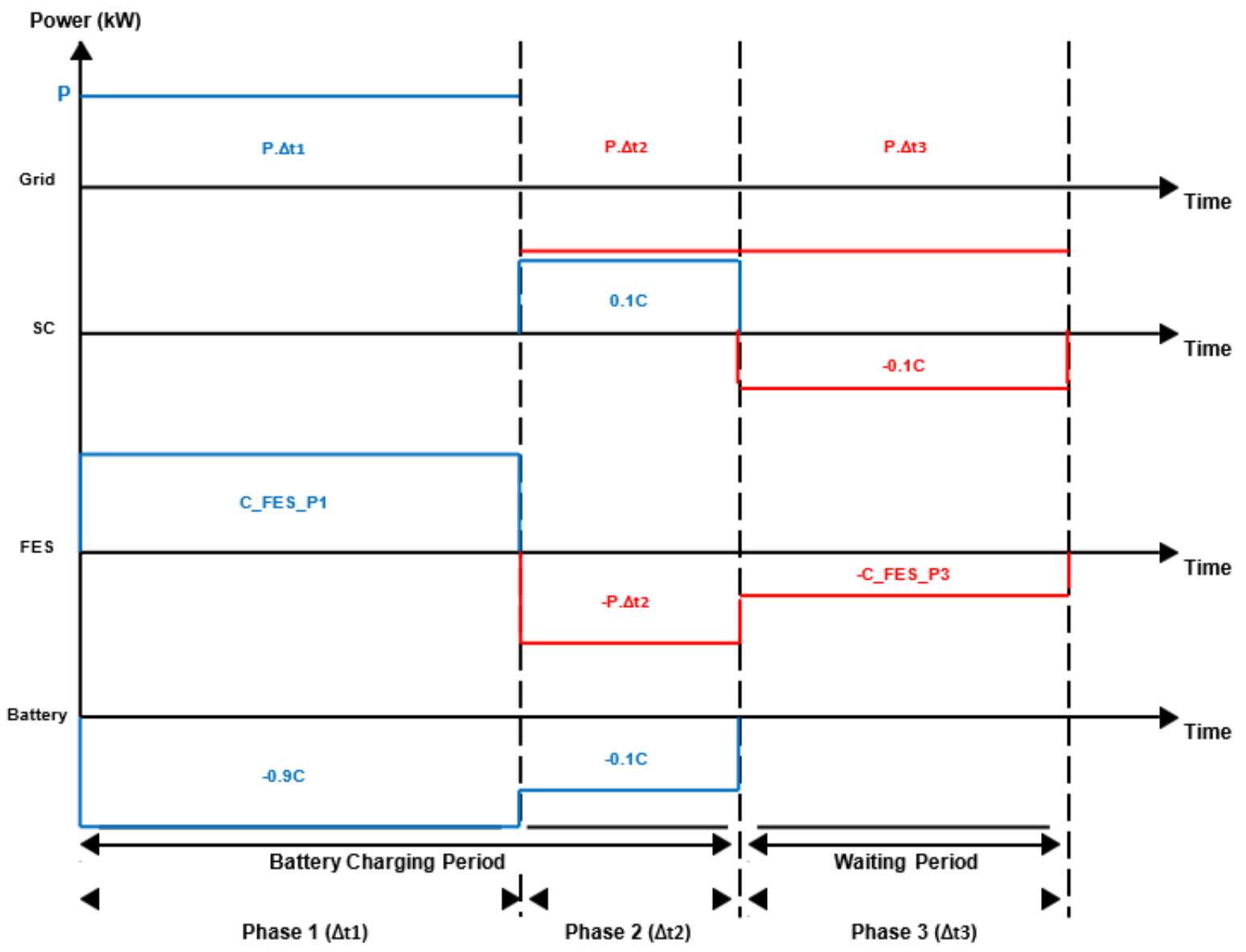

Fig.9: Detailed Charging Station Cycle

While the grid provides $5 \mathrm{kWh}$ of the maximum charging station output energy of $11.25 \mathrm{kWh}$ (see Table 2.3) during
Phase 1 , the storage devices provide the remaining $6.25 \mathrm{kWh}$ as follows: 
$1.125 \mathrm{kWh}$ is provided from the supercapacitor during Phase 2, and 5.125 $\mathrm{kWh}$ is provided from the FES during Phase 1.

The choice of such proportions is in accordance with the station's storage devices characteristics (see Section 2.3.2): the supercapacitor is in operation for a relatively small period (maximum of 5 minutes) compared to the FES operation duration (maximum 10 minutes).

Once a PHEV arrives at the charging station, the parameters in Figure that need to be computed are listed in Table 5.1 below.

Table.5.1: Optimization Parameters

\begin{tabular}{|l|c|c|c|c|c|}
\hline rameter Name & $\begin{array}{c}\text { Phase 1 Duration } \\
(\mathrm{min})\end{array}$ & $\begin{array}{c}\text { Phase 2 Duration } \\
(\mathrm{min})\end{array}$ & $\begin{array}{c}\text { Phase 3 Duration } \\
(\mathrm{min})\end{array}$ & $\begin{array}{l}\text { FES capacity in } \\
\text { phase 1 }(\mathrm{kWh})\end{array}$ & $\begin{array}{l}\text { FES capacity in } \\
\text { phase 3 }(\mathrm{kWh})\end{array}$ \\
\hline $\begin{array}{l}\text { Symbolic } \\
\text { Notation }\end{array}$ & $\Delta \mathrm{t}_{1}$ & $\Delta \mathrm{t}_{2}$ & $\Delta \mathrm{t}_{3}$ & CFES-P1 & CFES-P3 \\
\hline $\begin{array}{l}\text { Variable } \\
\text { Maxim um }\end{array}$ & 10 & 5 & 7.5 & 5.125 & 5.125 \\
\hline
\end{tabular}

- The maximum of each parameter is displayed in table 5.1 .

- As already mentioned, in Phase 1, the FES and the grid (who delivers a power, $\mathrm{p}$, in $\mathrm{kW}$ ) are recharging the PHEV battery to $90 \%$ of its required capacity, C.

p. $\Delta \mathrm{t}_{1}+\mathrm{C}_{\mathrm{FES}-\mathrm{P} 1=}$

0.9.C

(5.4)

- In Phase 3, the grid is recharging the supercapacitor and the FES:

p. $\Delta \mathrm{t}_{3}-\mathrm{C}_{\mathrm{FES}-\mathrm{P} 3}-0.1 \mathrm{C}=$

0

(5.5)

- The FES is providing energy to the PHEV battery during Phase 1, and is being recharged by the grid during Phases 2 and 3:

$\mathrm{C}_{\mathrm{FES}-\mathrm{P} 1}-\mathrm{p} . \Delta \mathrm{t}_{2}-\mathrm{C}_{\mathrm{FES}-\mathrm{P} 3}=$

0

\section{Charging Station Operation}

The charging station operates as shown in the flowchart of Figure

Energy management parameters are given in tables 5.3, 5.5A, and $5.5 \mathrm{~B}$, where:

- Blue and red numbers indicate whether energy is being transferred from the charger to the battery, or from the grid to the storage devices (to recharge them), respectively.

- Positive and negative quantities indicate whether energy is being delivered or absorbed by the device, respectively.

The following battery charging characteristics are displayed in Figures:

- Battery SOC (in \%)

- Battery current (in A)

- Battery voltage (in V)

The following charger characteristics are displayed in Figures:

- FES speed (in $\mathrm{rad} / \mathrm{s}$ )

- Grid current (in A)

- FES current (in A)

- Supercapacitor current (in A)

- Total current (in A): The sum of the grid, FES, and supercapacitor currents

- Supercapacitor voltage (in V)

\section{CONCLUSION}

\section{Summary}

In this thesis, the basic configuration used for the design and analysis of the fast charging station (including energy requirements and storage devices choice) has been described, the different power electronic interfaces have been designed, and the individual control schemes of each converter and the control and proposed algorithm of the whole charging station have been presented.

\section{Conclusions}

In this thesis, the design and simulation of a fast-charging station for PHEV batteries has been developed. Combination 
of a flywheel and a super capacitor as additional stationary storage devices is an excellent option since it inherently has four advantages, high energy density, high power density, charging and discharging times in the order of minutes, and environmentally friendly. The developed algorithm efficiently manages the three-station energy sources and allows the charging of PHEV batteries whose capacities are below 15 $\mathrm{kWh}$ in a maximum duration of 15 minutes from $20 \%$ to $95 \%$ of their state-of-charge and maximizes the waiting time (to recharge the storage devices) to 7.5 minutes when no PHEV is present at the station. Afterwards the station enters the standby mode, where the supercapacitor voltage remains constant and its current tends asymptotically to zero, while the flywheel rotates at constant speed. The reduction of the duration of the charging station operation will accelerate the battery recharging process in a battery swapping scheme.

\section{RECOMMENDATIONS FOR FUTURE WORK}

While a general control algorithm has been developed in this research to minimize the battery charging time and the duration required to charge the storage devices, more research could be conducted on the following topics:

- Optimization of the combination of flywheel and supercapacitor in terms of energy and power sizing.

- Impact of the battery charger on the power quality of the electric grid supply.

- Design of more efficient converter systems for the flywheel and super capacitor charging schemes.

\section{REFERENCES}

[1] Dickerman, L., \& Harrison, J. (2010). A New Car, a New Grid. IEEE Power And Energy Magazine, 8(2), 55-61. doi: $10.1109 /$ mpe.2009.935553

[2] Electric Vehicle Chargers Level 3 - Products and Applications - BTCPower (Broadband TelCom Power, Inc.). (2018). Retrieved from http://www.btcpower.com/products-andapplications/electric-vehicle-chargers-level-3/

[3] SAE International. (2001). Electric vehicle conductive charge coupler. Warrendale, $\mathrm{Pa}$.

[4] EV Solutions | Electric Vehicle Charging Products \& Services. (2018). Retrieved from https://www.evsolutions.com/

[5] GM to guarantee electric car batteries for eight years. (2010). Physics Today. doi: 10.1063/pt.5.024501

[6] Mengtian,L. (2017). The Electric Car Charging Strategy Based on the User's Intention and Its Optimization. Journal Of Automation And Control, 5(1), 16-19. doi: 10.12691/automation-5-1-3

[7] Oman, H. (1999). Making batteries last longer [for electric vehicles]. IEEE Aerospace And Electronic Systems Magazine, 14(9), 19-21. doi: 10.1109/62.793449

[8] Singh, S., Dhar, A., \& Agarwal, A. (2015). Technical feasibility study of butanol-gasoline blends for powering medium-duty transportation spark ignition engine. Renewable Energy, 76, 706-716. doi: 10.1016/j.renene.2014.11.095

[9] Thiringer, T., \& Haghbin, S. (2015). Power Quality Issues of a Battery Fast Charging Station for a Fully Electric Public Transport System in Gothenburg City. Batteries, 1(1), 22-33. doi: 10.3390/batteries 1010022

[10] Vorel, P., Cervinka, D., Prochazka, P., Toman, M., \& Martis, J. (2016). High Efficiency Fast-Chargers for Lead-Acid Batteries. ECS Transactions, 74(1), 23-30. doi: 10.1149/07401.0023ecst

[11] Wirasingha, S., \& Emadi, A. (2011). Pihef: Plug-In Hybrid Electric Factor. IEEE Transactions On Vehicular Technology, 60(3), 1279-1284. doi: $10.1109 /$ tvt.2011.2115263

[12] Zhou, J., \& Notten, P. (2008). Studies on the degradation of $\mathrm{Li}$-ion batteries by the use of microreference electrodes. Journal Of Power Sources, 177(2), 553-560. doi: 10.1016/j.jpowsour.2007.11.032

[13] Niresh J, Dr.Neelakrishanan, Muthu C, Sabareesh G, Saravanan P, Tharan Vikram S(2017).Mitigating Instability in Electric Drive Vehicles Due to Time Varying Delays with Optimised Controller. International Journal of Advanced Engineering Research and Science(ISSN : 2349-6495(P) | 24561908(O)),4(5), 010-017. http://dx.doi.org/10.22161/ijaers.4.5.3

[14] Amjadi, Z., \& Williamson, S. (2010). PowerElectronics-Based Solutions for Plug-in Hybrid Electric Vehicle Energy Storage and Management Systems. IEEE Transactions On Industrial Electronics, 57(2), 608-616. doi: 10.1109/tie.2009.2032195

[15] Mizumoto, I., Yoshii, Y., Yamamoto, K., \& Oguma, H. (2018). Lead-acid storage battery recovery systemusing on-off constant current charge and short-large discharge pulses. Electronics Letters, 54(12), 777-779. doi: 10.1049/el.2018.1079

[16] Nicholas, M., \& Tal, G. (2013). Dynamics of Workplace Charging for Plug-in Electric Vehicles: How Much is Needed and at What Speed?. World Electric Vehicle Journal, 6(4), 819-828. doi: 10.3390/wevj6040819

[17] Young-Joo Lee, Khaligh, A., \& Emadi, A. (2009). 
Advanced Integrated Bidirectional $\mathrm{AC} / \mathrm{DC}$ and $\mathrm{DC} / \mathrm{DC}$

Converter for Plug-In Hybrid Electric Vehicles. IEEE

Transactions On Vehicular Technology, 58(8), 3970-

3980. doi: 10.1109/tvt.2009.2028070

[18] P. Divya Sri, P., \& Prasad, D. (2011). Single Phase Dual Full Bridge Bi-directional DC-DC Converter for High power applications. Indian Journal Of Applied Research, 3(5), 259-265. doi: 10.15373/2249555x/may 2013/79 